\title{
RESEARCH
}

Open Access

\section{An MRI-based strategy for differentiation of frontotemporal dementia and Alzheimer's disease}

Qun Yu ${ }^{1 \dagger}$, Yingren Mai ${ }^{1 \dagger}$, Yuting Ruan ${ }^{1 \dagger}$, Yishan Luo ${ }^{2}$, Lei Zhao ${ }^{2}$, Wenli Fang ${ }^{1}$, Zhiyu Cao ${ }^{1}$, Yi Li $^{1}$, Wang Liao ${ }^{1}$, Songhua Xiao ${ }^{1}$, Vincent C. T. Mok ${ }^{2,3}$, Lin Shi ${ }^{2,4^{*}}$, Jun Liu ${ }^{1,5,6^{*}}$, the National Alzheimer's Coordinating Center, the Alzheimer's Disease Neuroimaging Initiative and the Frontotemporal Lobar Degeneration Neuroimaging Initiative

\begin{abstract}
Background: The differential diagnosis of frontotemporal dementia (FTD) and Alzheimer's disease (AD) is difficult due to the overlaps of clinical symptoms. Structural magnetic resonance imaging (sMRI) presents distinct brain atrophy and potentially helps in their differentiation. In this study, we aim at deriving a novel integrated index by leveraging the volumetric measures in brain regions with significant difference between AD and FTD and developing an MRI-based strategy for the differentiation of FTD and AD.
\end{abstract}

Methods: In this study, the data were acquired from three different databases, including 47 subjects with FTD, 47 subjects with AD, and 47 normal controls in the NACC database; 50 subjects with AD in the ADNI database; and 50 subjects with FTD in the FTLDNI database. The MR images of all subjects were automatically segmented, and the brain atrophy, including the AD resemblance atrophy index (AD-RAl), was quantified using AccuBrain ${ }^{\oplus}$. A novel MRI index, named the frontotemporal dementia index (FTDI), was derived as the ratio between the weighted sum of the volumetric indexes in "FTD dominant" structures over that obtained from "AD dominant" structures. The weights and the identification of "FTD/AD dominant" structures were acquired from the statistical analysis of NACC data. The differentiation performance of FTDI was validated using independent data from ADNI and FTLDNI databases.

Results: AD-RAl is a proven imaging biomarker to identify AD and FTD from NC with significantly higher values $(p<0.001$ and $A U C=0.88$ ) as we reported before, while no significant difference was found between AD and FTD $(p=0.647)$. FTDI showed excellent accuracy in identifying FTD from AD (AUC $=0.90 ; \mathrm{SEN}=89 \%, \mathrm{SPE}=75 \%$ with threshold value $=1.08)$. The validation using independent data from ADNI and FTLDNI datasets also confirmed the efficacy of FTDI (AUC = 0.93; SEN = $96 \%, \mathrm{SPE}=70 \%$ with threshold value $=1.08)$.

\footnotetext{
* Correspondence: shilin@cuhk.edu.hk; liujun6@mail.sysu.edu.cn

${ }^{\dagger}$ Qun Yu, Yingren Mai and Yuting Ruan contributed equally to this work. ${ }^{2}$ BrainNow Research Institute, Shenzhen, China

'Department of Neurology, Sun Yat-sen Memorial Hospital, Sun Yat-sen University, No. 107 Yanjiang West Road, Guangzhou, Guangdong, China Full list of author information is available at the end of the article
}

(c) The Author(s). 2021 Open Access This article is licensed under a Creative Commons Attribution 4.0 International License, which permits use, sharing, adaptation, distribution and reproduction in any medium or format, as long as you give appropriate credit to the original author(s) and the source, provide a link to the Creative Commons licence, and indicate if changes were made. The images or other third party material in this article are included in the article's Creative Commons licence, unless indicated otherwise in a credit line to the material. If material is not included in the article's Creative Commons licence and your intended use is not permitted by statutory regulation or exceeds the permitted use, you will need to obtain permission directly from the copyright holder. To view a copy of this licence, visit http://creativecommons.org/licenses/by/4.0/ The Creative Commons Public Domain Dedication waiver (http://creativecommons.org/publicdomain/zero/1.0/) applies to the data made available in this article, unless otherwise stated in a credit line to the data. 


\begin{abstract}
(Continued from previous page)
Conclusions: Brain atrophy in AD, FTD, and normal elderly shows distinct patterns. In addition to AD-RAl that is designed to detect abnormal brain atrophy in dementia, a novel index specific to FTD is proposed and validated. By combining AD-RAl and FTDI, an MRI-based decision strategy was further proposed as a promising solution for the differential diagnosis of AD and FTD in clinical practice.
\end{abstract}

Keywords: Frontotemporal dementia, Alzheimer's disease, Structural magnetic resonance imaging, AD resemblance atrophy index, Frontotemporal dementia index

\section{Introduction}

Frontotemporal dementia (FTD) is one of the main causes of dementia in people under 65, which accounts for nearly $20 \%$ of neurodegenerative dementia [1]. Clinical symptoms of FTD overlap with other types of dementia, psychiatric disorders, or Parkinson's disease. Alzheimer's disease (AD) is the most common type of dementia and is often difficult to differentiate with FTD, especially in the early stage $[2,3]$. Currently, there are no disease-modifying treatments for FTD. Acetylcholinesterase inhibitors widely used in patients with $\mathrm{AD}$ could lead to worsening of symptoms in those with FTD [4]. Therefore, accurate diagnosis of FTD and AD may avoid severe consequence in treatment outcome. In addition to clinical practice, the reduction of misdiagnosis is of utility in the differentiation for clinical trials, as well [5].

Structural magnetic resonance imaging (sMRI) is a common non-invasive examination in the diagnosis of dementia. Compared with computed tomography, positron emission tomography, and advanced MRI sequences, sMRI is advantageous in being widely accessible, free of ionizing or nuclear radiation, objective for visualizing brain atrophy, and with good tissue contrast [6]. In clinical practice, neurologists and radiologists use various visual rating scales to evaluate the degree of brain atrophy in MR images [7, 8]. In spite of being convenient to perform, visual rating is highly subjective and dependent on experience which is prone to lead to misdiagnosis and missed diagnosis in practice [9]. Recently, automatic brain segmentation, quantitative analysis, and machine learning have been studied a lot in neurodegenerative diseases [10-18]. However, plenty of machine learning-based classification highly rely on limited training data and lack of underlying clinical rationale [19-22]. Because it is hard for clinicians to understand how and why these artificial intelligence methods made a certain decision, the lack of interpretability limits their applicability in clinical practice [23]. Secondly, most studies were based on samples from a single center and may not be translated to other centers because of the heterogeneity in diagnostic criteria, imaging parameters, and sociodemographic characteristics of patients. Finally, many studies only focus on distinguishing $\mathrm{AD}$ from behavioral variant frontotemporal dementia (bvFTD) [24, 25]. But, in fact, due to the presence of either mild or overlapping linguistic deficits, $A D$ with speech impairment can also be confused with the subtypes of FTD, such as primary progressive aphasia (PPA) including semantic variant PPA (svPPA) and non-fluent variant PPA (nfvPPA) [26]. Therefore, it is theoretically desirable and practically meaningful if $\mathrm{AD}$ can be differentiated with FTD spectrum disorders.

The primary objective of this study is to investigate the MRI volumetric and atrophic patterns among FTD, AD, and normal controls (NC) collected from multicenter data. Based on these findings, the secondary objective is to derive an atrophy index that is specific to reflect FTD brain atrophy, named FTD index (FTDI). FTDI was an individualized index derived from brain regions with significant volume difference between FTD and AD, which were identified by group-level comparisons, and the differentiation performance of the proposed index was validated using multicenter data from another two databases.

\section{Materials and methods Subjects}

In this retrospective study, all the subjects were included from the National Alzheimer's Coordinating Center (NACC), the Alzheimer's Disease Neuroimaging Initiative (ADNI), and the Frontotemporal Lobar Degeneration Neuroimaging Initiative (FTLDNI) databases (see Additional Material 1). The data are the result of collaborative efforts at three sites in North America.

We first included 47 subjects with FTD (19 bvFTD, 12 svPPA, 2 nfvPPA, and 14 not otherwise specified) from NACC. Then, 47 subjects with probable AD and $47 \mathrm{NC}$ perfectly matching those with FTD (Table 1 ) in age, educational level, gender, race, and global CDR score (except for $\mathrm{NC}$ ) using the $\mathrm{CDR}^{\circ}$ Dementia Staging Instrument plus NACC FTLD Behavior \& Language Domains [2729] were selected from NACC as well. Subjects were included if their diagnoses were stable ( $>1$ year) and 3D T1weighted MRI scans were available \pm 6 months of a visit. Scans containing large artifacts were excluded by visual inspection (Fig. 1). After that, as a validation dataset, 50 subjects with $A D$ in the ADNI database and 50 subjects with FTD (20 bvFTD, 10 svPPA, 10 nfvPPA, and 10 not otherwise specified) in the FTLDNI database were included in this study. 
Table 1 Demographic characteristics of subjects included

\begin{tabular}{|c|c|c|c|c|}
\hline NACC & FTD $(n=47)$ & $\mathrm{AD}(n=47)$ & NC $(n=47)$ & $p$ value \\
\hline Age (years), mean (SD) & $64.55(10.15)$ & $64.55(10.28)$ & $64.57(10.13)$ & $>0.999$ \\
\hline Education (years), mean (SD) & $15.45(2.98)$ & $15.62(2.78)$ & $15.68(2.86)$ & 0.920 \\
\hline Gender, male $(n(\%))$ & $25(53.2)$ & $25(53.2)$ & $25(53.2)$ & $>0.999$ \\
\hline Race, White (n (\%)) & $47(100.0)$ & $47(100.0)$ & $47(100.0)$ & $>0.999$ \\
\hline ICV $(\mathrm{mL})$, mean $(\mathrm{SD})$ & $1472.14(155.82)$ & $1526.37(170.79)$ & $1505.12(145.51)$ & 0.247 \\
\hline Global CDR^ & & & & $>0.999$ \\
\hline $0.0=$ no impairment $(n(\%))$ & $6(12.8)$ & $6(12.8)$ & $47(100.0)$ & - \\
\hline $0.5=$ questionable impairment $(n(\%))$ & $21(44.7)$ & $21(44.7)$ & - & - \\
\hline $1.0=$ mild impairment $(n(\%))$ & $17(36.2)$ & $17(36.2)$ & - & - \\
\hline $2.0=$ moderate impairment $(n(\%))$ & $3(6.4)$ & $3(8.5)$ & - & - \\
\hline ADNI + FTLDNI & FTD $(n=50)$ & $\mathrm{AD}(n=50)$ & - & $p$ value \\
\hline Age (years), mean (SD) & $64.64(7.09)$ & $64.22(7.58)$ & - & 0.484 \\
\hline Education (years), mean (SD) & $15.98(3.57)$ & $16.02(2.56)$ & - & 0.241 \\
\hline Gender, male $(n(\%))$ & $27(54.0)$ & $23(46.0)$ & - & 0.548 \\
\hline Race, White $(n(\%))$ & $45(90.0)$ & $46(92.0)$ & - & 0.727 \\
\hline ICV $(\mathrm{mL})$, mean $(\mathrm{SD})$ & $1521.82(131.45)$ & $1456.48(158.80)$ & & 0.070 \\
\hline Global CDR^ & & & - & 0.002 \\
\hline $0.0=$ no impairment $(n(\%))$ & $3(6.0)$ & $0(0.0)$ & - & - \\
\hline $0.5=$ questionable impairment $(n(\%))$ & $24(48.0)$ & $20(40.0)$ & - & - \\
\hline $1.0=$ mild impairment $(n(\%))$ & $16(32.0)$ & $30(60.0)$ & - & - \\
\hline $2.0=$ moderate impairment $(n(\%))$ & $7(14.0)$ & $0(0.0)$ & - & - \\
\hline
\end{tabular}

$\wedge$ Data was compared between the FTD and AD groups only. $p$ value would be highlighted in bold when it was below 0.05

The National Institute of Neurological and Communicative Disorders and Stroke and the $\mathrm{AD}$ and Related Disorders Association (NINCDS/ADRDA) criteria [30] and the National Institute on Aging-Alzheimer's Association (NIA-AA) criteria [31] were applied for the diagnosis of probable AD. The Mckhann et al. and Rascovsky et al. criteria [32, 33] were used for bvFTD, and the Mckhann et al. and Gorno-Tempini et al. criteria [32, 34] for PPA. The inclusion criteria for the NC were normal in general physical status and CDR of 0 , suggesting no cognitive impairment.

\section{MRI acquisition and volumetric analysis}

MR images from NACC were collected following varying acquisition protocols by different centers, which reflects the real-world scenario. In the ADNI database, the subjects were scanned using 3-T scanners including GE, Philips, or Siemens scanners. For T1-weighted MRI, the

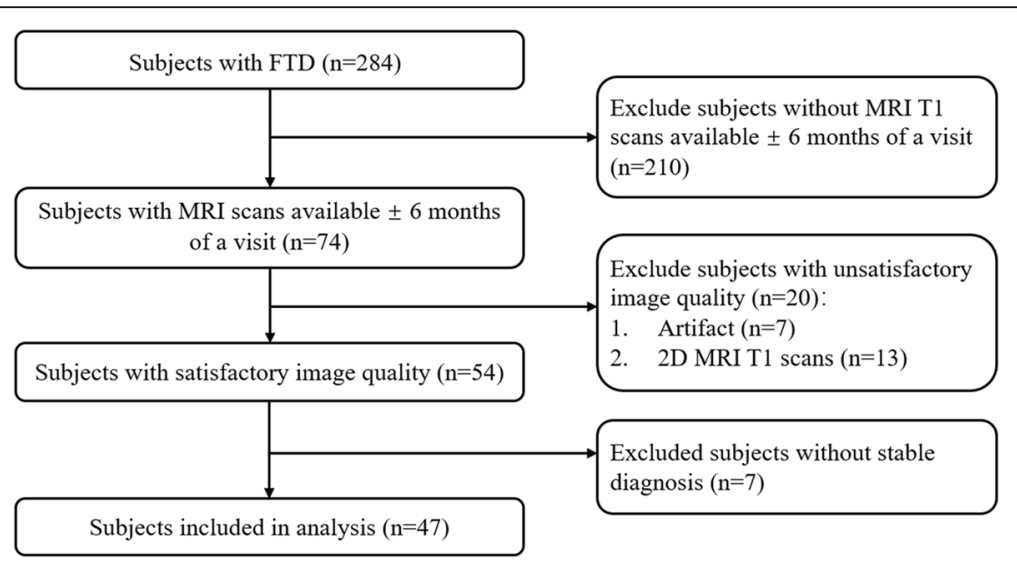

Fig. 1 Subject screening from the NACC database 
inversion recovery-fast spoiled gradient recalled (IRFSPGR) sequences were used by GE scanners, and magnetization-prepared rapid gradient echo (MP-RAGE) sequences were used by Philips and Siemens scanners. More details concerning the sMRI images are available on the ADNI homepage (http://adni.loni.usc.edu/ methods/mri-tool/mri-analysis/). In the FTLDNI database, the subjects were scanned using Siemens TrioTim 3-T scanners at three centers. MP-RAGE sequence was used to acquire T1-weighted MR images [35]. All MRI scans were analyzed using AccuBrain (BrainNow Medical Technology Limited), a fully automatic neuroanatomical volumetry tool that quantifies brain volumes of various subcortical structures, ventricles, and cortical lobar atrophy within clinically acceptable time. AccuBrain ${ }^{\circ}$ performs brain structure segmentation based on a multi-atlas image registration scheme. The accuracy of hippocampus segmentation of AccuBrain ${ }^{\bullet}$ was validated using data from the ADNI database [36], and more technical description of AccuBrain ${ }^{\circ}$ and its comparison with other automatic brain image segmentation schemes were described in our previous work [37-39]. Since the main objective of this study is to identify FTD from NC and $\mathrm{AD}$, we focus on the brain regions known to be associated with cognition and behavior, including the brain parenchyma, typical subcortical structures (bilateral hippocampus, amygdala and caudate, etc.), ventricular regions (ventricular system, lateral ventricle, and inferior lateral ventricle), and atrophy of lobar regions (frontal, occipital, temporal, parietal, cingulate, and insular lobe). To correct for the head size difference, we took the relative volume and atrophy as our volumetric brain measures. The relative volume of each region was defined as the absolute volume divided by the total intracranial volume (ICV). The atrophy of a particular lobe was defined as the ratio between the volume of cerebrospinal fluid and brain parenchyma within that lobe. The AD resemblance atrophy index (AD-RAI), representing the similarity of the brain atrophy pattern with $\mathrm{AD}$, was also calculated for each subject in this study. Calculating ADRAI is a unique function of AccuBrain and was previously proven to be able to identify $\mathrm{AD}$ and estimate the risk of cognitive decline [40, 41].

In addition to AD-RAI, in this study, a novel MRIbased biomarker named FTDI was derived based on the volumetric results of brain regions with significant volume difference between $\mathrm{AD}$ and $\mathrm{FTD}$ using the NACC data.

To increase the comparability of the numerical metrics, the relative volumes of the involved brain regions and lobe atrophy $R_{i}(j)$ were normalized to $R^{\prime}{ }_{i}(j)$, i.e.,

$$
R_{i}^{\prime}(j)=\frac{R_{i}(j)-\min \left(R_{i}\right)}{\max \left(R_{i}\right)-\min \left(R_{i}\right)}
$$

where $R_{i}(j)$ is the relative volume of the $i$ th volumetric brain measure of the $j$ th subject among all the subjects from NACC included in this study. For the ease of formulation, assume values in the array $R_{i}$ are arranged so that the first $m$ values are the subjects with FTD and the total number of subjects with FTD and AD is $n$. The "FTD dominant" structures are the structures with statistically larger $R_{i}$ values in the FTD group, and the "AD dominant" structures are those with statistically larger $R_{i}$ values in the AD group. It should be noted that the "FTD/AD dominant" structures did not refer to the abnormal brain regions of FTD/AD patients. They were derived from the group-level comparisons and were the volumetric metrics with the statistically larger values. $\min \left(R_{i}\right)$ and $\max \left(R_{i}\right)$ represent the minimal and maximal values in the $i$ th volumetric brain measure of all subjects from NACC included in this study, respectively.

As each lobe atrophy contributed uniquely in the differentiation of $\mathrm{AD}$ and FTD, a weight $w_{i}$ was calculated for each "FTD dominant" and "AD dominant" structures as the normalized absolute difference of $R_{i}$ between FTD and AD patients:

$$
w_{i}=\frac{\left|\sum_{j=1}^{m} R_{i}(j)-\sum_{j=m+1}^{n} R_{i}(j)\right|}{\sum_{j=1}^{m} R_{i}(j)+\sum_{j=m+1}^{n} R_{i}(j)}
$$

In order to increase the differentiation ability, the FTDI of an individual brain is defined as the ratio between its weighted sum of $R_{i}$ in the "FTD dominant" structures over that of the "AD dominant" structures.

$$
\operatorname{FTDI}(j)=\frac{\sum_{i=1}^{p} w_{i} R_{i}^{\prime}(j)}{\sum_{i=p+1}^{q} w_{i} R_{i}^{\prime}(j)}
$$

where $i=\{1, \ldots, p\}$ are the "FTD dominant" structures and $i=\{p+1, \ldots, q\}$ are the "AD dominant" structures.

When FTDI was validated using the ADNI and FTLD NI data, the maximum and minimum values from NACC were used in the normalization and any value of $R_{i}{ }^{\prime}(j)$ below zero would be set to zero.

\section{Visual assessment}

All the MRI scans were viewed in the coronal plane and the severity of medial temporal lobe atrophy (MTA) was assessed visually by two experienced neurologists (YRM and YTR) according to a 5-point scale [42]. The single MTA score of each subject was the average score of left and right hemispheres with 
substantial agreement and high reliability between the two raters $(\kappa=0.75$, ICC $>0.9)$.

\section{Statistical analysis}

We compared the demographic characteristics of the three groups (NC, AD, FTD) using ANOVA analysis and chi-square test. Data of volumetric brain measures were not normally distributed and therefore were analyzed statistically with the Friedman $M$ test, and a $p$ value below 0.05 was considered to indicate a significant difference. If significant differences were found, a post hoc analysis would be performed with the Wilcoxon signed-rank test with Bonferroni correction, and a corrected $p$ value below 0.05 was considered to indicate a significant difference. Receiver operating characteristic (ROC) curves were drawn, and the area under the curve (AUC), sensitivity (SEN), and specificity (SPE) were used to verify the diagnostic efficiency of AD-RAI and FTDI. Nonnormally distributed values were described as medians with inter-quartile range. All the statistical analyses were performed with software (SPSS version 25.0, IBM, Armonk, NY).

\section{Results}

\section{Demographic and clinical parameters}

The characteristics (age, educational level, gender, race, and global CDR score) of subjects from NACC were matched in pairs and the changes among each group showed no significant difference (Table 1). The characteristics of subjects from ADNI and FTLDNI were also displayed (Table 1).

\section{AD-RAl can identify AD and FTD from NC}

Compared with the $\mathrm{NC}$ group, we found that the $\mathrm{AD}$ group showed significant $(p<0.05)$ atrophy in the brain parenchyma, bilateral hippocampi, temporal lobes, parietal lobes, amygdalae, and left cingulate lobe, and the ventricular system was significantly enlarged (see Additional Table 1). The FTD group showed significant $(p<$ 0.05 ) atrophy in the brain parenchyma, bilateral frontal lobes, temporal lobes, insular lobes, amygdalae, and left cingulate lobe (see Additional Table 1). These findings are largely consistent with previous studies [43-45]. The AD-RAI was significantly different between NC when compared with $\mathrm{AD}$ and FTD, respectively (Table 2, Fig. 2a). As we expected, AD-RAI performed well (AUC $=0.88$ ) with $97.9 \%$ sensitivity and $73.4 \%$ specificity (Fig. 3a). However, when comparing FTD with AD, ADRAI showed no significant $(p=0.647)$ difference (Table 2 , Fig. 2a).

\section{Diagnostic performance of single volumetric brain measure and FTDI}

We found that the relative volume of the total hippocampus, left caudate, left frontal lobe, right occipital lobe, bilateral parietal lobes, bilateral insular lobes, and atrophy of bilateral frontal lobes and right occipital lobe were significantly $(p<0.05)$ different (Table 2 , Fig. $2 \mathrm{~b}-\mathrm{l})$

Table 2 Comparison of AD-RAl and volumetric brain measures among NC, AD, and FTD groups from NACC

\begin{tabular}{|c|c|c|c|c|c|c|}
\hline & \multirow[t]{2}{*}{$\mathrm{NC}(n=47)$} & \multirow[t]{2}{*}{$\mathrm{AD}(n=47)$} & \multirow{2}{*}{$\begin{array}{l}\text { FTD }(n= \\
47)\end{array}$} & \multicolumn{3}{|l|}{$p$ value } \\
\hline & & & & NC vs $A D$ & NC vs FTD & AD vs FTD \\
\hline AD-RAI & $0.12(0.20)$ & $0.94(0.51)$ & $0.97(0.23)$ & $<0.001$ & $<0.001$ & 0.647 \\
\hline Hippocampus & $0.45(0.05)$ & $0.39(0.09)$ & $0.42(0.07)$ & $<0.001$ & 0.446 & 0.008 \\
\hline Caudate (L) & $0.23(0.02)$ & $0.23(0.04)$ & $0.21(0.04)$ & 0.491 & 0.836 & 0.040 \\
\hline Frontal lobe (L) atrophy & $42.50(14.40)$ & $42.30(13.30)$ & $51.30(14.10)$ & $>0.999$ & $<0.001$ & 0.002 \\
\hline Frontal lobe (R) atrophy & $40.70(14.80)$ & $43.40(12.80)$ & $51.50(15.30)$ & 0.647 & $<0.001$ & 0.001 \\
\hline Occipital lobe (R) atrophy & $9.11(4.82)$ & $12.70(5.90)$ & $10.00(5.46)$ & $<0.001$ & $>0.999$ & $<0.001$ \\
\hline Frontal lobe $(\mathrm{L})$ & $5.56(0.67)$ & $5.50(0.51)$ & $5.22(0.65)$ & $>0.999$ & 0.001 & 0.022 \\
\hline Occipital lobe (R) & $2.26(0.31)$ & $2.13(0.52)$ & $2.36(0.34)$ & $>0.999$ & 0.150 & 0.022 \\
\hline Temporal lobe (L) & $3.71(0.32)$ & $3.30(0.40)$ & $3.06(0.76)$ & $<0.001$ & $<0.001$ & 0.061 \\
\hline Temporal lobe (R) & $3.68(0.37)$ & $3.30(0.55)$ & $3.20(0.71)$ & $<0.001$ & $<0.001$ & 0.540 \\
\hline Parietal lobe $(\mathrm{L})$ & $2.63(0.31)$ & $2.42(0.38)$ & $2.63(0.38)$ & $<0.001$ & $>0.999$ & $<0.001$ \\
\hline Parietal lobe (R) & $2.81(0.42)$ & $2.50(0.37)$ & $2.86(0.40)$ & 0.005 & 0.239 & $<0.001$ \\
\hline Insular (L) & $0.47(0.05)$ & $0.42(0.05)$ & $0.36(0.07)$ & 0.239 & $<0.001$ & $<0.001$ \\
\hline Insular (R) & $0.48(0.05)$ & $0.46(0.05)$ & $0.42(0.09)$ & 0.103 & $<0.001$ & 0.012 \\
\hline QMTA & $0.33(0.07)$ & $0.55(0.32)$ & $0.52(0.38)$ & $<0.001$ & $<0.001$ & $>0.999$ \\
\hline MTA score & $0.25(1.00)$ & $1.50(1.50)$ & $1.50(1.50)$ & $<0.001$ & $<0.001$ & $>0.999$ \\
\hline
\end{tabular}

The comparison was performed with Friedman $M$ test and a post hoc analysis with Wilcoxon signed-rank test with Bonferroni correction. $p$ value would be highlighted in bold when it was below 0.05 . The median with inter-quartile range (in bracket) of AD-RAl and the volumetric brain measures in all three groups were provided. $L$ left, $R$ right 

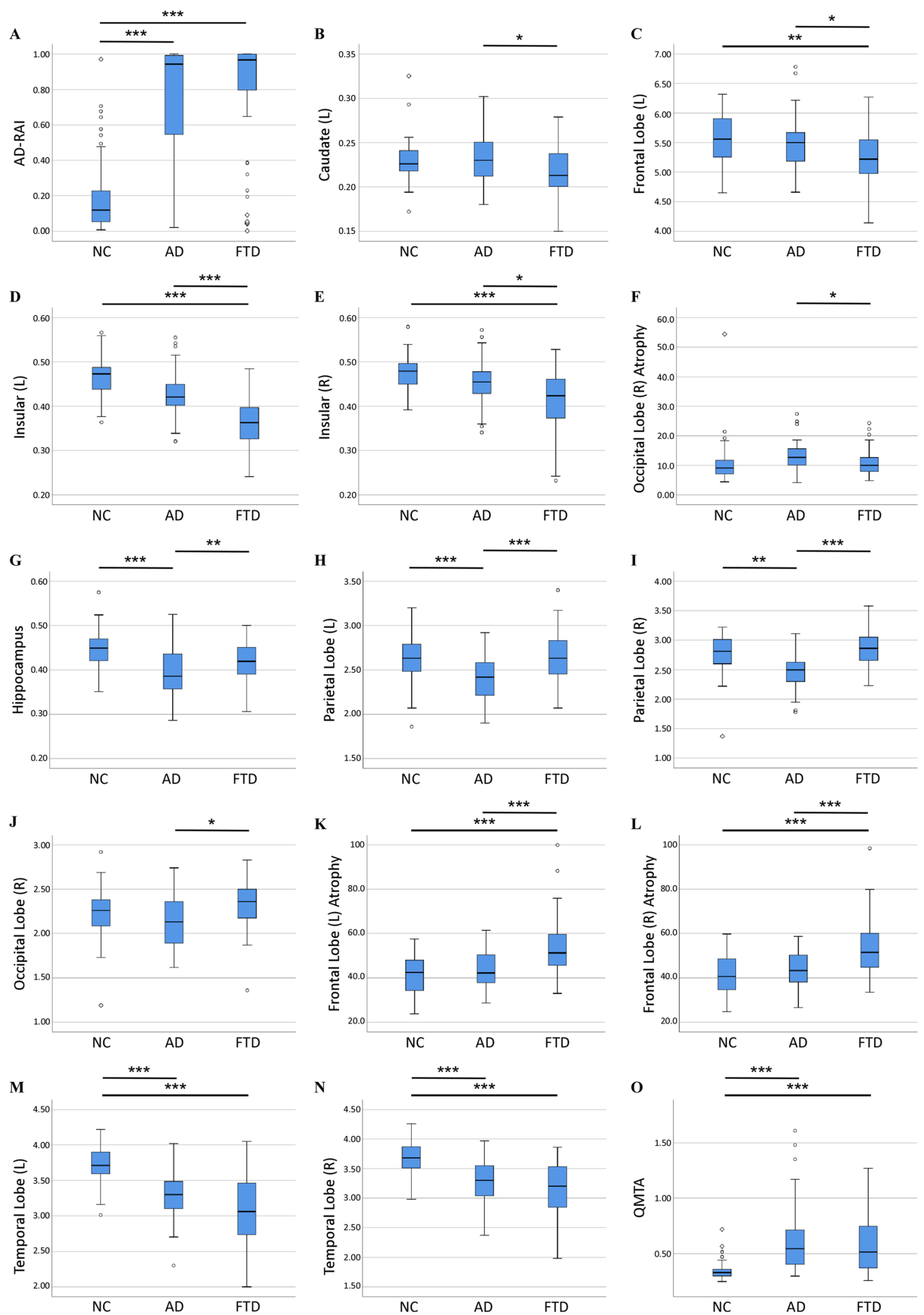

Fig. 2 Box plots of AD-RAl and volumetric brain measures among NC, AD, and FTD groups from the NACC database. a AD-RAl among NC, AD, and FTD groups. b-f Volumetric brain measures which were significantly lower in the FTD group than in AD. $\mathbf{g}-\mathbf{I}$ Volumetric brain measures which were significantly higher in the FTD group than in AD. $\mathbf{m}, \mathbf{n}$ Relative volumes of bilateral temporal lobes and $\mathbf{o}$ QMTA among NC, AD, and FTD groups $\left({ }^{*} p<0.05,{ }^{* *} p<0.01,{ }^{* * *} p<0.001\right)$ 

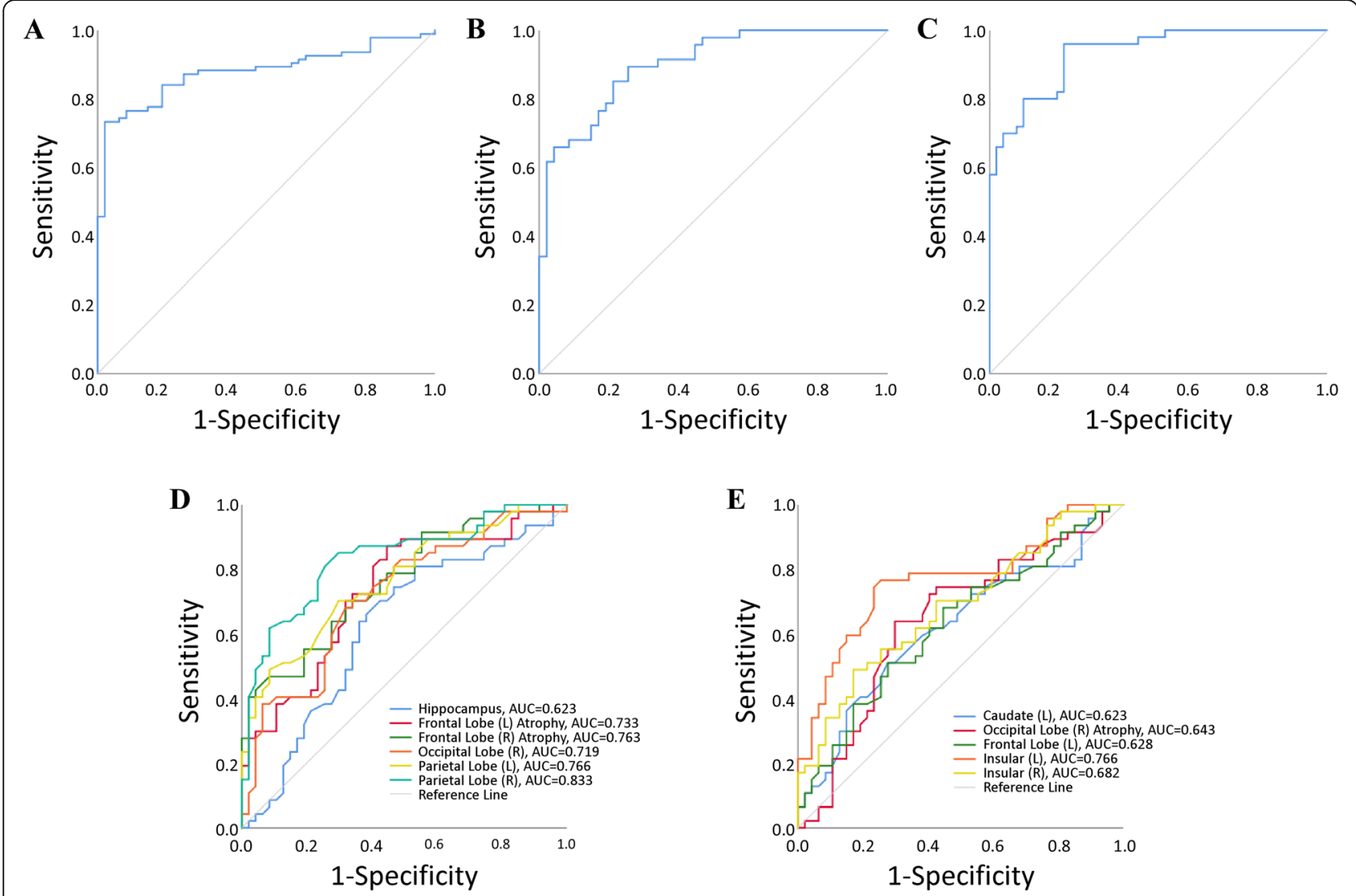

Fig. 3 Diagnostic performance of different indexes. a ROC of AD-RAl using data from the NACC database, AUC $=0.88$. $\mathbf{b}$ ROC of FTDI using data from the NACC database, $A \cup C=0.90$. $\mathbf{c}$ ROC of FTDI using data from ADNI and FTDNI databases, $A \cup C=0.93$. $\mathbf{d}$, e ROC of the single volumetric brain measure which was significantly different between AD and FTD groups

between $\mathrm{AD}$ and FTD groups. However, the temporal lobe, MTA score, and quantitative MTA (QMTA, defined as the ratio between the volumes of the inferior lateral ventricle and hippocampus using AccuBrain and Spearmen correlation of QMTA and MTA score was 0.805 [40]) of AD and FTD all showed significant difference with normal people but no significant difference existed between the two in this study (Table 2, Fig. 2mo). As previously mentioned, among these structures, those with statistically larger volumetric brain measure in the FTD group were defined as the "FTD dominant" structures and those with statistically larger volumetric brain measure in the AD group were defined as the "AD dominant" structures, and the ratio between the two derived FTDI. When a single volumetric brain measure was used to distinguish FTD from $\mathrm{AD}$ in the NACC database, the AUC could only reach 0.63 0.83 (Fig. 3d, e). The parietal lobe, consistent with the previous study [43], provided the highest diagnostic accuracy. When FTDI was used for differential diagnosis of FTD and AD in the NACC database, the AUC could reach 0.90 (Fig. 3b). We chose FTDI $>1.08$ as the threshold value according to the optimal Youden Index, which ensures high sensitivity (89.4\%) and relatively good specificity (74.5\%). When validated in the data from ADNI and FTDNI databases, FTDI performed robustly with $96.0 \%$ sensitivity and $70.0 \%$ specificity (AUC $=0.93$, Fig. $3 \mathrm{c}$ ).

\section{Discussion}

In this study, we developed a novel MRI biomarker for differentiating AD and FTD using their dominant brain volumetric measures derived from statistical analysis of the automated and objective volumetry. The CDR scores of subjects with FTD/AD included from NACC in our study ranged mostly from 0 to 1 (>93\%) and MTA scores using visual rating scale showed no significant difference between the two groups, which means most of them experienced an early stage of $\mathrm{AD} / \mathrm{FTD}$ and the atrophy patterns of the two may overlap or be too subtle (Fig. 4). Group-level comparisons were firstly applied to find the distinct brain atrophy patterns between FTD and AD (Fig. 5), and FTDI was exactly an individual index derived from those differential volumetric brain measures. By combining with the previously developed 


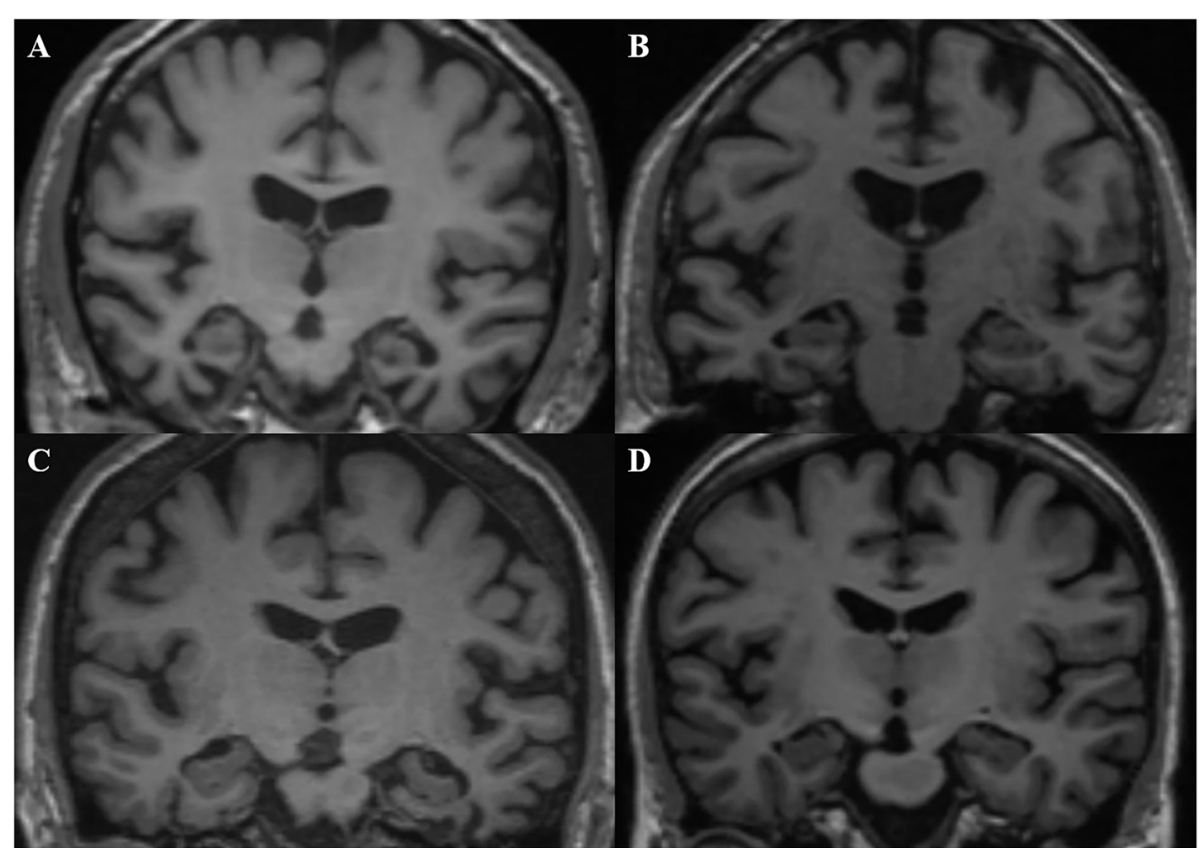

Fig. 4 3D T1-weighted MRI scans (classical coronal section) of a FTD with CDR =0.5, ID NACC204983; b AD with CDR =0.5, ID NACC162576; $\mathbf{c}$ FTD with $C D R=1.0$, ID NACC067187; and $\mathbf{d}$ AD with CDR = 1.0, ID NACC878860

AD-RAI, an MRI-based diagnostic strategy was designed (Fig. 6) for the differentiation of FTD and AD. The FTDI model and the threshold value was determined using data from NACC and was validated in a separate cohort of data combined from ADNI and FTDNI databases. The accuracy of AD-RAI in identifying the AD and FTD from $\mathrm{NC}$ measured by AUC is 0.88 . In this strategy, we set the threshold value of 0.75 with high sensitivity (97.9\%) to reduce the rate of missed diagnosis, while maintaining a relatively high specificity (73.4\%). When distinguishing FTD from AD, the AUC of FTDI was 0.90 , which is comparable and even higher than previous studies [19-22, 46, 47]. The advantage of FTDI is that it is intuitively interpretable with a clear clinical meaning. Unlike complicated machine learning method or advanced imaging examinations, our work based on sMRI provided a simple logical algorithm and decision strategy to help clinicians understand and use. As the therapeutic strategy needs to be tailored for FTD and AD, the high accuracy in differentiating FTD and AD is more favored. For example, cholinesterase inhibitors effective against AD may aggravate the condition of FTD patients [4]. With this consideration, we chose a threshold value of FTDI of 1.08 with high sensitivity $(89.4 \%)$ to reduce potential missed diagnosis of FTD. When validated on the independent testing data from ADNI and FTDNI databases, FTDI was proven to be satisfactorily accurate (AUC $=0.93, \mathrm{SEN}=96 \%$. Our research was based on real-world data analysis and hence has high interpretability.

The ideal imaging-assisted diagnosis for clinical use should be objective, economic, simple, and efficient. Therefore, many studies adopted the automatic brain segmentation tool to identify FTD and AD through machine learning. These models need to be trained on a large number of high-quality and uniformly acquired images. Obtaining these images from a population with a low incidence of FTD will be a challenge. In addition, these methods are considered to be the "black box" and difficult for clinicians to understand, so most of them are hard to be adopted in clinical practice. In this study, we acquired enough subjects from three different wellrecognized multicenter databases to ensure the robustness of the results that can be translated to the realworld application. Secondly, AccuBrain ${ }^{\odot}$ is a National Medical Products Administration (NMPA) and CEmarked commercially automated brain segmentation tool that can be readily deployed in the clinic. Thirdly, clinicians without computer expertise can easily apply FTDI to daily clinical practice. Thus, compared with most existing MRI-based FTD classification methods, FTDI has a higher clinical application prospect.

A few studies have attempted to classify the subtypes of FTD based on MRI [20, 46, 47]. The proposed methodology in this study could be further extended into the classification of FTD subtypes. But it is beyond the scope 


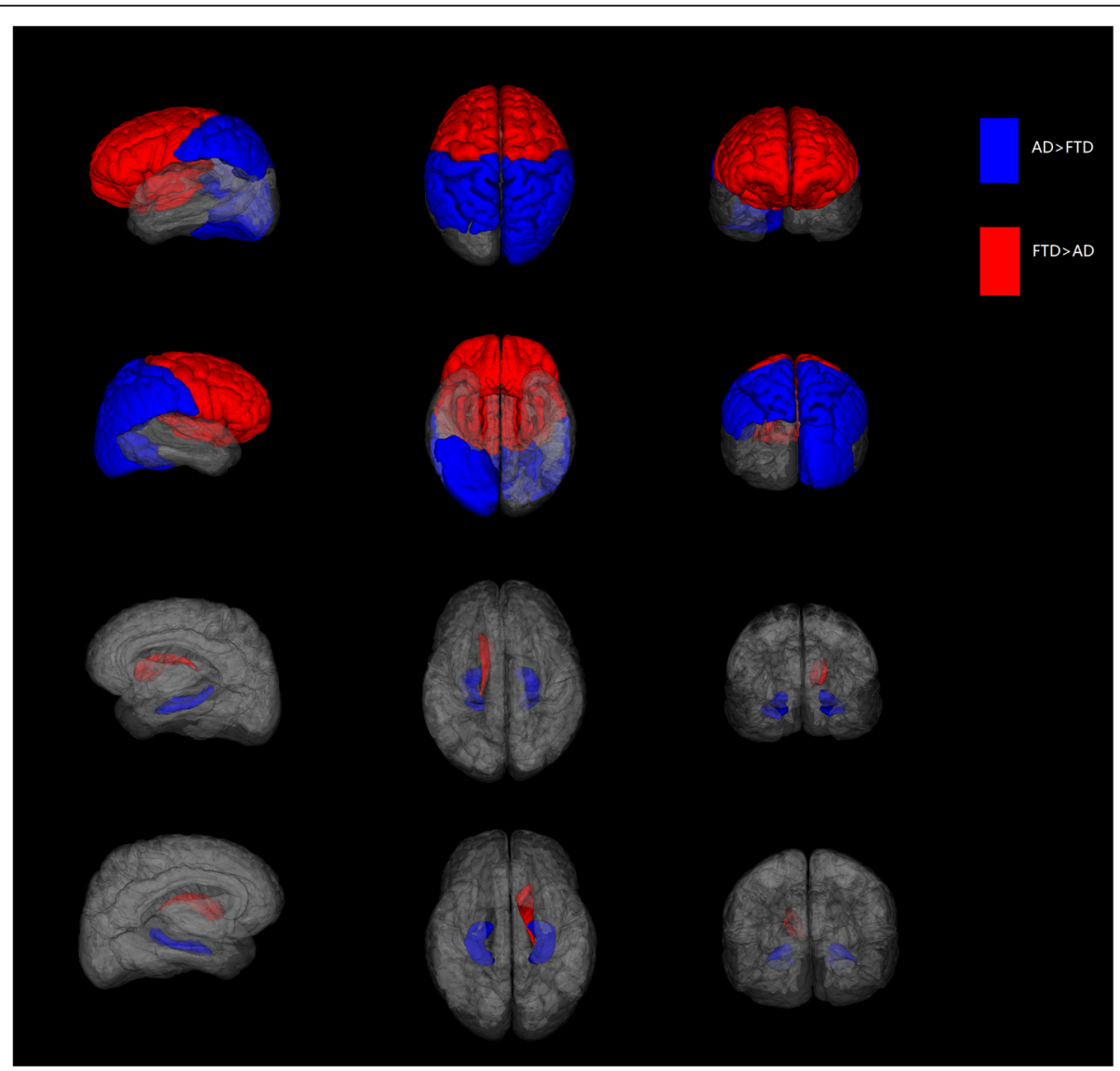

Fig. 5 The distinct brain atrophy patterns between FTD and AD. The red color labeled the brain structures with higher atrophy in FTD including the bilateral frontal lobes, bilateral insular lobes, and left caudate. Likewise, the blue color labeled the brain structures with higher atrophy in AD including the bilateral parietal lobes, right occipital lobe, and total hippocampi

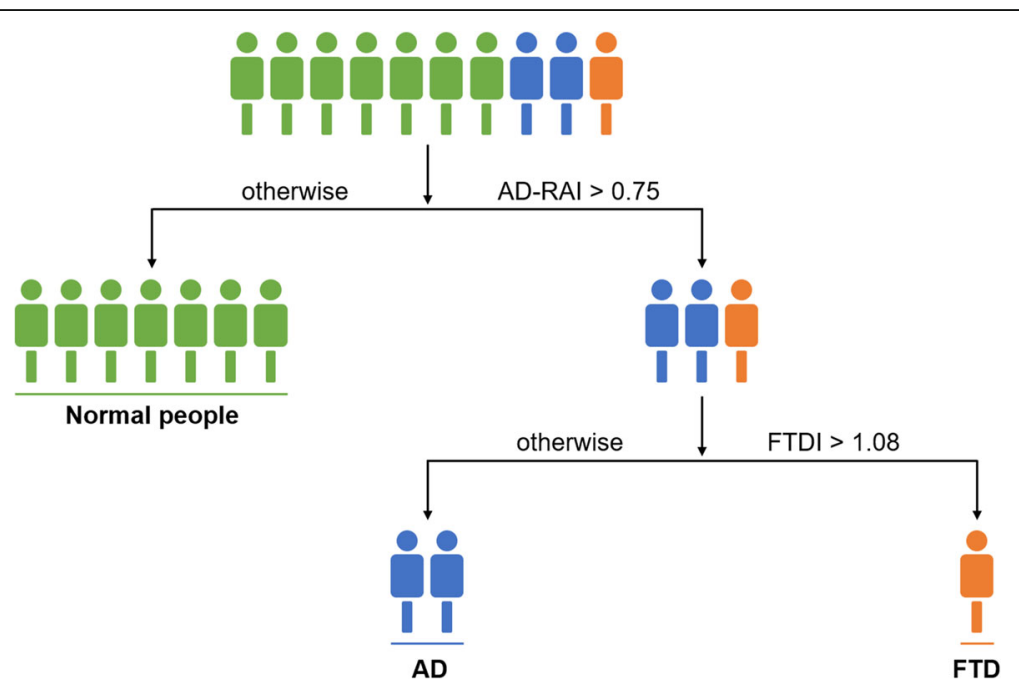

Fig. 6 Quantitative evidence-based decision strategy to differentially diagnose AD and FTD from NC 
of this study. This study, instead, is focused on a more important clinical problem, which is the differentiation of FTD as a whole with AD. However, different proportion of subtypes of the FTD spectrum disorders may result in a potential patient selection bias. FTDI is a composite index derived from several different volumetric brain measures after weighted. Preliminary validation in this work has proven its good robustness.

\section{Limitations}

This study has several limitations. First of all, pathological diagnosis is not mandatory during subject inclusion, although it may reduce the likelihood of misdiagnosis. However, essentially, there is no recognized in vivo pathological biomarker of FTD [48, 49] and the pathological diagnosis of FTD usually comes from autopsy, which increases the difficulty of acquisition. To ensure adequate sample size, we relied more on the clinical diagnosis as indicated in the database to include the subjects. Another limitation of this study is that it was only tested and validated on three databases. Although inclusion and exclusion criteria of these databases were fairly standard and comparable to those in clinical trials, the CDR status is not reflective of the distribution of analogous patients seen in clinical practice and could bias results. However, the results of this work preliminarily showed the benefits of FTDI and our decision strategy in differentiation of FTD and AD. In addition, this study included 241 subjects in total, which is a relatively modest sample size. Before application to clinical settings, a larger dataset for further test and validation is needed. It can be difficult to collect data and maintain a relatively consistent standard, which may have a significant effect on MRI analysis. Meanwhile, due to the current limited data, the diagnostic efficacy of FTDI is unproven for patients beyond the age range specified in this study.

Although this study has these limitations, it provides a novel algorithm that takes full advantage of the different patterns of brain atrophy in AD and FTD, and it can incorporate more data to make the diagnosis more accurate. Most importantly, through this study, we came up with a method to derive individualized FTDI and provided its threshold value using the data from the NACC database and developed an MRI-based decision strategy, which is obviously more beneficial for clinicians to understand and use than complex machine learning programs.

\section{Conclusions}

In this study, the volumetric metrics of structural MRI were investigated in a multicenter cohort of FTD, AD, and NC. These volumetric features and group-level difference were then explored to an FTD atrophy-specific index, named FTDI. Combined with the AD resemblance atrophy index, AD-RAI, we developed a strategy for identifying and differentiating FTD and AD based on MRI-induced volumetric information. Compared with visual rating and machine learning, the proposed strategy is more accurate, objective, and easier to implement.

\section{Supplementary Information}

The online version contains supplementary material available at https://doi. org/10.1186/s13195-020-00757-5.

Additional file 1: Additional Material 1. Brief introduction of the databases where data were collected.

Additional file 2: Additional Table 1. Comparison of all the volumetric brain measures among NC, AD and FTD group from NACC. The comparison was performed with Friedman $M$ test and a post hoc analysis with Wilcoxon signed-rank test with Bonferroni correction. $P$ value would be highlighted in bold when it was below 0.05 and would not be shown if difference was not significant among the three groups. The median with inter-quartile range of AD-RAI and the volumetric brain measures in all three groups were provided. $L$, left; $R$, right.

\section{Abbreviations}

AD: Alzheimer's disease; FTD: Frontotemporal dementia; sMRI: Structural magnetic resonance imaging; bvFTD: Behavioral variant frontotemporal dementia; PPA: Primary progressive aphasia; svPPA: Semantic variant PPA; nfvPPA: Non-fluent variant PPA; NC: Normal controls; NACC: National Alzheimer's Coordinating Center; ADNI: Alzheimer's Disease Neuroimaging Initiative; FTLDNI: Frontotemporal Lobar Degeneration Neuroimaging Initiative; AD-RAI: AD resemblance atrophy index; FTDI: Frontotemporal dementia index; MCl: Mild cognitive impairment; CDR: Clinical dementia rating; ICV: Intracranial volume; MTA: Medial temporal atrophy; ICC: Interclass correlation coefficient; ROC curve: Receiver operating characteristic curve; AUC: Area under the curve; SEN: Sensitivity; SPE: Specificity

\section{Acknowledgements}

We are grateful for the research volunteers, their families, and the investigators at the NACC, ADNI, and FTLDNI databases. The NACC database is funded by National Institutes on Aging/National Institutes of Health (NIA/ NIH) Grant U01 AG016976. NACC data are contributed by the NIA-funded ADCs: P30 AG019610 (PI Eric Reiman, MD), P30 AG013846 (PI Neil Kowall, MD), P30 AG062428-01 (PI James Leverenz, MD), P50 AG008702 (PI Scott Small, MD), P50 AG025688 (PI Allan Levey, MD, PhD), P50 AG047266 (PI Todd Golde, MD, PhD), P30 AG010133 (PI Andrew Saykin, PsyD), P50 AG005146 (PI Marilyn Albert, PhD), P30 AG062421-01 (PI Bradley Hyman, MD, PhD), P30 AG062422-01 (PI Ronald Petersen, MD, PhD), P50 AG005138 (PI Mary Sano, PhD), P30 AG008051 (PI Thomas Wisniewski, MD), P30 AG013854 (PI Robert Vassar, PhD), P30 AG008017 (PI Jeffrey Kaye, MD), P30 AG010161 (PI David Bennett, MD), P50 AG047366 (PI Victor Henderson, MD, MS), P30 AG010129 (PI Charles DeCarli, MD), P50 AG016573 (PI Frank LaFerla, PhD), P30 AG062429-01 (PI James Brewer, MD, PhD), P50 AG023501 (PI Bruce Miller, MD), P30 AG035982 (PI Russell Swerdlow, MD), P30 AG028383 (PI Linda Van Eldik, PhD), P30 AG053760 (PI Henry Paulson, MD, PhD), P30 AG010124 (PI John Trojanowski, MD, PhD), P50 AG005133 (PI Oscar Lopez, MD), P50 AG005142 (PI Helena Chui, MD), P30 AG012300 (PI Roger Rosenberg, MD), P30 AG049638 (PI Suzanne Craft, PhD), P50 AG005136 (PI Thomas Grabowski, MD), P30 AG062715-01 (PI Sanjay Asthana, MD, FRCP), P50 AG005681 (PI John Morris, MD), and P50 AG047270 (PI Stephen Strittmatter, MD, PhD). ADNI data collection and sharing for this project was funded by the NIH Grant U01 AG024904 and DOD ADNI (Department of Defense award number W81XWH-12-2-0012). ADNI is funded by the National Institute on Aging, the National Institute of Biomedical Imaging and Bioengineering, and through generous contributions from the following: AbbVie, Alzheimer's Association; Alzheimer's Drug Discovery Foundation; Araclon Biotech; BioClinica, Inc.; Biogen; Bristol-Myers Squibb Company; CereSpir, Inc.; Cogstate; Eisai Inc.; Elan Pharmaceuticals, Inc.; Eli Lilly and Company; Eurolmmun; F. Hoffmann-La Roche Ltd. and its affiliated company Genentech, Inc.; Fujirebio; GE 
Healthcare; IXICO Ltd.; Janssen Alzheimer Immunotherapy Research \& Development, LLC.; Johnson \& Johnson Pharmaceutical Research \& Development LLC.; Lumosity; Lundbeck; Merck \& Co., Inc.; Meso Scale Diagnostics, LLC.; NeuroRx Research; Neurotrack Technologies; Novartis Pharmaceuticals Corporation; Pfizer Inc.; Piramal Imaging; Servier; Takeda Pharmaceutical Company; and Transition Therapeutics. The Canadian Institutes of Health Research is providing funds to support ADNI clinical sites in Canada. Private sector contributions are facilitated by the Foundation for the National Institutes of Health (www.fnih.org). The grantee organization is the Northern California Institute for Research and Education, and the study is coordinated by the Alzheimer's Therapeutic Research Institute at the University of Southern California. ADNI data are disseminated by the Laboratory for Neuro Imaging at the University of Southern California.

FTLDNI data collection and sharing for this project was funded by the $\mathrm{NIH}$ Grant R01 AG032306. The study is coordinated through the University of California, San Francisco, Memory and Aging Center. FTLDNI data are disseminated by the Laboratory for Neuro Imaging at the University of Southern California.

Partial data used in the preparation of this article were obtained from the Alzheimer's Disease Neuroimaging Initiative (ADNI) database (adni.loni.usc. edu). As such, the investigators within the ADNI contributed to the design and implementation of ADNI and/or provided data but did not participate in the analysis or writing of this report. A complete listing of ADNI investigators can be found at http://adni.loni.usc.edu/wp-content/uploads/how_to_apply/ ADNI_Acknowledgement_List.pdf

Partial data used in the preparation of this article were obtained from the Frontotemporal Lobar Degeneration Neuroimaging Initiative (FTLDNI) database. The investigators at NIFD/FTLDNI contributed to the design and implementation of FTLDNI and/or provided data, but did not participate in the analysis or writing of this report (unless otherwise listed). The FTLDNI investigators included the following individuals:

Howard Rosen; University of California, San Francisco (PI)

Bradford C. Dickerson; Harvard Medical School and Massachusetts General Hospital

Kimoko Domoto-Reilly; University of Washington School of Medicine

David Knopman; Mayo Clinic, Rochester

Bradley F. Boeve; Mayo Clinic Rochester

Adam L. Boxer; University of California, San Francisco

John Kornak; University of California, San Francisco

Bruce L. Miller; University of California, San Francisco

William W. Seeley; University of California, San Francisco

Maria-Luisa Gorno-Tempini; University of California, San Francisco

Scott McGinnis; University of California, San Francisco

Maria Luisa Mandelli; University of California, San Francisco

\section{Authors' contributions}

QY designed the study, sorted the data, did the statistical analysis, and drafted and revised the manuscript. YRM and YTR scored MTA, interpreted the data, prepared all the figures and tables, and drafted and revised the manuscript. YSL, LZ, WLF, ZYC, YL, and WL sorted and inspected the data and revised the manuscript. SHX, VCM, LS, and JL interpreted the data and revised the manuscript. All authors contributed to the writing and revisions of the paper and approved the final version. Data used in this study were obtained from the NACC, ADNI, and FTLDNI. The investigators within the three provided data but were uninvolved in the data analysis or drafting of this manuscript.

\section{Funding}

This study was funded by the National Natural Science Foundation of China (grant numbers 81870836, 81872261) and the Natural Science Foundation of Guangdong Province (grant number 2020A1515010210). The funding body had no role in the design of the study and collection, analysis, interpretation of data, and writing of the manuscript.

\section{Availability of data and materials}

The datasets used and analyzed during the current study are available from the corresponding author on reasonable request.

\section{Ethics approval and consent to participate}

This study was approved by the institutional review boards of all participating institutions, and written informed consent was obtained from all participants or their guardians according to the Declaration of Helsinki. A complete listing of ADNI investigators and institutions can be found online (http://adni.loni.usc.edu/wp-content/uploads/how_to_apply/ADNI_ Acknowledgement_List.pdf).

\section{Consent for publication}

Not applicable.

\section{Competing interests}

LS is the director of BrainNow Medical Technology Limited. VCM is the chief medical consultant of BrainNow Medical Technology Limited. YSL and LZ are now employed by BrainNow Medical Technology Limited. All other authors report no financial relationships with commercial interests.

\section{Author details}

${ }^{1}$ Department of Neurology, Sun Yat-sen Memorial Hospital, Sun Yat-sen University, No. 107 Yanjiang West Road, Guangzhou, Guangdong, China. ${ }^{2}$ BrainNow Research Institute, Shenzhen, China. ${ }^{3}$ Gerald Choa Neuroscience Centre, Lui Che Woo Institute of Innovative Medicine, Division of Neurology, Department of Medicine and Therapeutics, The Chinese University of Hong Kong, Hong Kong SAR, China. ${ }^{4}$ Department of Imaging and Interventional Radiology, The Chinese University of Hong Kong, Hong Kong SAR, China.

${ }^{5}$ Guangdong Province Key Laboratory of Brain Function and Disease, Zhongshan School of Medicine, Sun Yat-sen University, Guangzhou, China. ${ }^{6}$ Laboratory of RNA and Major Diseases of Brain and Heart, Sun Yat-sen Memorial Hospital, Sun Yat-sen University, Guangzhou, China.

Received: 11 September 2020 Accepted: 21 December 2020 Published online: 12 January 2021

\section{References}

1. Ratnavalli E, Brayne C, Dawson K, Hodges JR. The prevalence of frontotemporal dementia. Neurology. 2002;58(11):1615-21.

2. Liscic RM, Storandt M, Cairns NJ, Morris JC. Clinical and psychometric distinction of frontotemporal and Alzheimer dementias. Arch Neurol. 2007; 64(4):535-40.

3. Seo SW, Thibodeau MP, Perry DC, Hua A, Sidhu M, Sible I, Vargas JNS, Gaus SE, Rabinovici GD, Rankin KD, et al. Early vs late age at onset frontotemporal dementia and frontotemporal lobar degeneration. Neurology. 2018;90(12): e1047-56.

4. Mendez MF, Shapira JS, McMurtray A, Licht E. Preliminary findings: behavioral worsening on donepezil in patients with frontotemporal dementia. Am J Geriat Psychiatry. 2007;15(1):84-7.

5. Wolz R, Schwarz AJ, Gray KR, Yu P, Hill DL. Enrichment of clinical trials in $\mathrm{MCl}$ due to $\mathrm{AD}$ using markers of amyloid and neurodegeneration. Neurology. 2016;87(12):1235-41.

6. Young PNE, Estarellas M, Coomans E, Srikrishna M, Beaumont H, Maass A, Venkataraman AV, Lissaman R, Jiménez D, Betts MJ, et al. Imaging biomarkers in neurodegeneration: current and future practices. Alzheimers Res Ther. 2020;12(1):49.

7. Falgàs N, Sánchez-Valle R, Bargalló N, Balasa M, Fernández-Villullas G, Bosch B, Olives J, Tort-Merino A, Antonell A, Muñoz-García C, et al. Hippocampal atrophy has limited usefulness as a diagnostic biomarker on the early onset Alzheimer's disease patients: a comparison between visual and quantitative assessment. Neurolmage Clin. 2019;23:101927.

8. Galton CJ, Gomez-Anson B, Antoun N, Scheltens P, Patterson K, Graves M, Sahakian BJ, Hodges JR. Temporal lobe rating scale: application to Alzheimer's disease and frontotemporal dementia. J Neurol Neurosurg Psychiatry. 2001;70(2):165-73.

9. Falgàs N, Balasa M, Bargalló N, Borrego-Écija S, Ramos-Campoy O, Fernández-Villullas G, Bosch B, Olives J, Tort-Merino A, Antonell A, et al. Diagnostic accuracy of MRI visual rating scales in the diagnosis of early onset cognitive impairment. J Alzheimers Dis. 2020;73(4):1575-83.

10. Meyer S, Mueller K, Stuke K, Bisenius S, Diehl-Schmid J, Jessen F, Kassubek J, Kornhuber J, Ludolph AC, Prudlo J, et al. Predicting behavioral variant frontotemporal dementia with pattern classification in multi-center structural MRI data. Neurolmage Clin. 2017;14:656-62.

11. Salvatore C, Battista P, Castiglioni I. Frontiers for the early diagnosis of AD by means of MRI brain imaging and support vector machines. Curr Alzheimer Res. 2016;13(5):509-33. 
12. Wolf D, Bocchetta M, Preboske GM, Boccardi M, Grothe MJ. Reference standard space hippocampus labels according to the European Alzheimer's Disease Consortium-Alzheimer's Disease Neuroimaging Initiative harmonized protocol: utility in automated volumetry. Alzheimer's Dement. 2017;13(8):893-902.

13. Vernooij MW, Jasperse B, Steketee $R$, Koek M, Vrooman H, Ikram MA, Papma J, van der Lugt A, Smits M, Niessen WJ. Automatic normative quantification of brain tissue volume to support the diagnosis of dementia: a clinical evaluation of diagnostic accuracy. Neurolmage Clin. 2018;20:374-9.

14. Tong T, Wolz R, Gao Q, Guerrero R, Hajnal JV, Rueckert D. Multiple instance learning for classification of dementia in brain MRI. Med Image Anal. 2014; 18(5):808-18.

15. Zhu X, Suk HI, Lee SW, Shen D. Subspace regularized sparse multitask learning for multiclass neurodegenerative disease identification. IEEE Trans Biomed Eng. 2016;63(3):607-18.

16. Chagué P, Marro B, Fadili S, Houot M, Morin A, Samper-González J, Beunon $P$, Arrivé L, Dormont D, Dubois B, et al. Radiological classification of dementia from anatomical MRI assisted by machine learning-derived maps. J Neuroradiol. 2020. https://doi.org/10.1016/j.neurad.2020.04.004.

17. Wenzel F, Meyer C, Stehle T, Peters J, Siemonsen S, Thaler C, Zagorchev L. Rapid fully automatic segmentation of subcortical brain structures by shape-constrained surface adaptation. Med Image Anal. 2018;46:146-61.

18. Ansart M, Epelbaum S, Bassignana G, Bône A, Bottani S, Cattai T, Couronné R, Faouzi J, Koval I, Louis M, et al. Predicting the progression of mild cognitive impairment using machine learning: a systematic, quantitative and critical review. Med Image Anal. 2020;67:101848.

19. Bron EE, Smits M, Papma JM, Steketee RME, Meijboom R, de Groot M, van Swieten JC, Niessen WJ, Klein S. Multiparametric computer-aided differential diagnosis of Alzheimer's disease and frontotemporal dementia using structural and advanced MRI. Eur Radiol. 2017;27(8):3372-82

20. Klöppel S, Peter J, Ludl A, Pilatus A, Maier S, Mader I, Heimbach B, Frings L, Egger K, Dukart J, et al. Applying automated MR-based diagnostic methods to the memory clinic: a prospective study. J Alzheimers Dis. 2015;47(4):939-54.

21. Dukart J, Mueller K, Horstmann A, Barthel H, Möller HE, Villringer A, Sabri O, Schroeter ML. Combined evaluation of FDG-PET and MRI improves detection and differentiation of dementia. PLoS One. 2011:6(3):e18111.

22. Raamana PR, Rosen H, Miller B, Weiner MW, Wang L, Beg MF. Three-class differential diagnosis among Alzheimer disease, frontotemporal dementia, and controls. Front Neurol. 2014;5:71.

23. Kelly CI, Karthikesalingam A, Suleyman M, Corrado G, King D. Key challenges for delivering clinical impact with artificial intelligence. BMC Med. 2019;17(1):195.

24. Möller C, Pijnenburg YA, van der Flier WM, Versteeg A, Tijms B, de Munck JC, Hafkemeijer A, Rombouts SA, van der Grond J, van Swieten J, et al. Alzheimer disease and behavioral variant frontotemporal dementia: automatic classification based on cortical atrophy for single-subject diagnosis. Radiology. 2016;279(3):838-48.

25. Möller C, Hafkemeijer A, Pijnenburg YA, Rombouts SA, van der Grond J, Dopper E, van Swieten J, Versteeg A, Pouwels PJ, Barkhof F, et al. Joint assessment of white matter integrity, cortical and subcortical atrophy to distinguish AD from behavioral variant FTD: a two-center study. Neurolmage Clin. 2015;9:418-29.

26. Harris JM, Gall C, Thompson JC, Richardson AM, Neary D, du Plessis D, Pal P, Mann DM, Snowden JS, Jones M. Classification and pathology of primary progressive aphasia. Neurology. 2013;81(21):1832-9.

27. Premi E, Gualeni V, Costa P, Cosseddu M, Gasparotti R, Padovani A, Borroni B. Looking for measures of disease severity in the frontotemporal dementia continuum. J Alzheimers Dis. 2016;52(4):1227-35.

28. Miyagawa T, Brushaber D, Syrjanen J, Kremers W, Fields J, Forsberg LK, Heuer HW, Knopman D, Kornak J, Boxer A, et al. Utility of the global CDR( $\left.{ }^{\oplus}\right)$ plus NACC FTLD rating and development of scoring rules: data from the ARTFL/LEFFTDS Consortium. Alzheimer's Dement. 2020;16(1):106-17.

29. Miyagawa T, Brushaber D, Syrjanen J, Kremers W, Fields J, Forsberg LK, Heuer HW, Knopman D, Kornak J, Boxer A, et al. Use of the CDR ${ }^{\oplus}$ plus NACC FTLD in mild FTLD: data from the ARTFL/LEFFTDS consortium. Alzheimer's Dement. 2020;16(1):79-90.

30. McKhann G, Drachman D, Folstein M, Katzman R, Price D, Stadlan EM. Clinical diagnosis of Alzheimer's disease: report of the NINCDS-ADRDA Work Group under the auspices of Department of Health and Human Services Task Force on Alzheimer's Disease. Neurology. 1984;34(7):939-44.

31. McKhann GM, Knopman DS, Chertkow H, Hyman BT, Jack CR Jr, Kawas CH, Klunk WE, Koroshetz WJ, Manly JJ, Mayeux R, et al. The diagnosis of dementia due to Alzheimer's disease: recommendations from the National Institute on Aging-Alzheimer's Association workgroups on diagnostic guidelines for Alzheimer's disease. Alzheimer's Dement. 2011;7(3):263-9.

32. McKhann GM, Albert MS, Grossman M, Miller B, Dickson D, Trojanowski JQ. Clinical and pathological diagnosis of frontotemporal dementia: report of the Work Group on Frontotemporal Dementia and Pick's Disease. Arch Neurol. 2001;58(11):1803-9.

33. Rascovsky K, Hodges JR, Knopman D, Mendez MF, Kramer JH, Neuhaus J, van Swieten JC, Seelaar H, Dopper EG, Onyike CU, et al. Sensitivity of revised diagnostic criteria for the behavioural variant of frontotemporal dementia. Brain. 2011;134(Pt 9):2456-77.

34. Gorno-Tempini ML, Hillis AE, Weintraub S, Kertesz A, Mendez M, Cappa SF, Ogar JM, Rohrer JD, Black S, Boeve BF, et al. Classification of primary progressive aphasia and its variants. Neurology. 2011;76(11):1006-14.

35. Manera AL, Dadar M, Collins DL, Ducharme S. Deformation based morphometry study of longitudinal MRI changes in behavioral variant frontotemporal dementia. Neurolmage Clin. 2019;24:102079.

36. Abrigo J, Shi L, Luo Y, Chen Q, Chu WCW, Mok VCT. Standardization of hippocampus volumetry using automated brain structure volumetry tool for an initial Alzheimer's disease imaging biomarker. Acta Radiol (Stockholm). 2019;60(6):769-76.

37. Gao L, Liu L, Shi L, Luo Y, Wang Z, Guo X, Xing B. Dynamic changes of views on the brain changes of Cushing's syndrome using different computer-assisted tool. Rev Endocr Metab Disord. 2020;21(1):185-200.

38. Zhao W, Luo Y, Zhao L, Mok V, Su L, Yin C, Sun Y, Lu J, Shi L, Han Y. Automated brain MRI volumetry differentiates early stages of Alzheimer's disease from normal aging. J Geriatr Psychiatry Neurol. 2019;32(6):354-64.

39. Wang W, Wong L, Shi L, Luo Y, Liang Z, Dong C, Song Q, Liu T, Zhang Q, Liu A, et al. Association of impaired fasting glucose and type 2 diabetes mellitus with brain volume changes in Alzheimer's disease patients analyzed by MRI: a retrospective study. PeerJ. 2020;8:e9801.

40. Zhao L, Liu W, Luo Y, Kung WK, Xiong L, Mok V, Shi L. Visual rating and automated brain volumetry for medial temporal lobe atrophy in differential diagnosis and early detection of Alzheimer's disease. In: Alzheimer's Association International Conference; 2020.

41. Zhao L, Luo Y, Lew D, Liu W, Au L, Mok V, Shi L. Risk estimation before progression to mild cognitive impairment and Alzheimer's disease: an AD resemblance atrophy index. Aging. 2019;11(16):6217-36.

42. Scheltens $P$, Leys $D$, Barkhof $F$, Huglo D, Weinstein HC, Vermersch $P$, Kuiper M, Steinling M, Wolters EC, Valk J. Atrophy of medial temporal lobes on MRI in "probable" Alzheimer's disease and normal ageing: diagnostic value and neuropsychological correlates. J Neurol Neurosurg Psychiatry. 1992;55(10): 967-72.

43. Du AT, Schuff N, Kramer JH, Rosen HJ, Gorno-Tempini ML, Rankin K, Miller $B L$, Weiner MW. Different regional patterns of cortical thinning in Alzheimer's disease and frontotemporal dementia. Brain. 2007;130(Pt 4): 1159-66.

44. Rosen HJ, Gorno-Tempini ML, Goldman WP, Perry RJ, Schuff N, Weiner M, Feiwell R, Kramer JH, Miller BL. Patterns of brain atrophy in frontotemporal dementia and semantic dementia. Neurology. 2002;58(2):198-208.

45. Pini L, Pievani M, Bocchetta M, Altomare D, Bosco P, Cavedo E, Galluzzi S, Marizzoni M, Frisoni GB. Brain atrophy in Alzheimer's disease and aging. Ageing Res Rev. 2016;30:25-48.

46. Kim JP, Kim J, Park YH, Park SB, Lee JS, Yoo S, Kim EJ, Kim HJ, Na DL, Brown $J A$, et al. Machine learning based hierarchical classification of frontotemporal dementia and Alzheimer's disease. Neurolmage Clin. 2019; 23:101811.

47. Bruun M, Koikkalainen J, Rhodius-Meester HFM, Baroni M, Gjerum L, van Gils M, Soininen H, Remes AM, Hartikainen P, Waldemar G, et al. Detecting frontotemporal dementia syndromes using MRI biomarkers. Neurolmage Clin. 2019;22:101711.

48. Hodges JR, Piguet O. Progress and challenges in frontotemporal dementia research: a 20-year review. J Alzheimers Dis. 2018;62(3):1467-80.

49. Meeter LH, Kaat LD, Rohrer JD, van Swieten JC. Imaging and fluid biomarkers in frontotemporal dementia. Nat Rev Neurol. 2017;13(7):406-19.

\section{Publisher's Note}

Springer Nature remains neutral with regard to jurisdictional claims in published maps and institutional affiliations. 